\title{
Engineering cavity modes in photonic crystal double-heterostructures
}

\author{
S. Mahmoodian ${ }^{1}$, K.B. Dossou ${ }^{2}$, C.G. Poulton ${ }^{2}$, R.C. McPhedran ${ }^{1}$, \\ L.C. Botten ${ }^{2}$ and C. M. De Sterke ${ }^{1}$ \\ Centre for Ultra-high bandwidth Devices for Optical Applications (CUDOS) \\ ${ }^{1}$ School of Physics, University of Sydney, Australia \\ ${ }^{2}$ Department of Mathematical Sciences, University of Technology, Sydney, Australia \\ E-mail: sahand@physics.usyd.edu.au,Chris.Poulton@uts.edu.au
}

\begin{abstract}
We present results from a new method that allows us to design the mode fields for 3D photonic crystal heterostructure cavities, in the domain where the perturbation used to create the cavity is weak. The method, based on a perturbation treatment from solid-state physics, enables the rapid computation of the main features of 3D cavity modes for an arbitrary perturbation and is several orders of magnitude faster than direct numerical methods. We use this method to study optimal confinement of resonant states in these structures. (C2010 Optical Society of America OCIS codes: (230.3990) (230.5750)
\end{abstract}

The efficient confinement of light in optical microcavities has emerged as one of the most important applications of photonic crystal slabs. In particular, photonic crystal double-heterostructure cavities (Fig. 1) are very promising because the Q-factors of these structures can be made to be very high; for this reason they are attractive to researchers seeking to enhance nonlinear interactions, and they have been proposed for use in a range of applications including all-optical switching and low-threshold lasers [1,2]. The flexibility of these structures has been underlined by the recent demonstration of the feasibility of obtaining high-Q resonators via an optically-induced change in the refractive index [3].

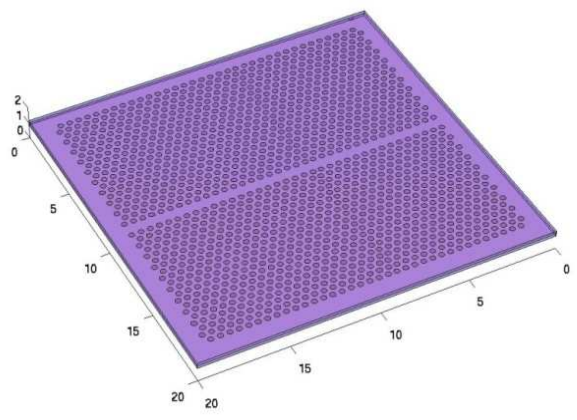

Figure 1: The geometry of a $3 D$ photonic crystal heterostructure considered here. A weak perturbation to the photonic crystal waveguide mode induces a cavity mode localized at the centre of the perturbation.

An important tool in achieving these high-Q cavity designs is tailoring of the envelope of the cavity mode: For a high-Q cavity one aims to have a quickly-decaying function in real space and at the same minimize the overlap between the confined mode and the light-cone: in this way the power lost to the boundaries of the photonic crystal and to free-space will be minimized. The ideal combination is one of "gentle confinement" that results in a Gaussian profile for the envelope of the cavity mode [2]. This strategy is however qualitative rather than quantitative, and so direct numerical computations must be employed to verify that the intended result is achieved. However, such calculations are extremely computationally expensive - a single computation sufficiently reliable for experimental design can take many hundreds of CPU hours. For weaker perturbations, which are typically required for ultrahigh quality factors and occur for optically-induced cavities, the situation is more dire - the resonance spreads out a long way from the perturbation centre and so a proportionally large computational domain is necessary for the field's accurate calculation. In these situations the accurate computation of the resonant fields is almost beyond the reach of direct numerical methods such as FDTD.

We present here results from a new method for cavity mode design for 3D heterostructure cavities, based on a semi-analytic model valid in the regime where the perturbation to the refractive index is small. The method is based on the framework of Luttinger and Kohn from the Solid-State Physics literature [4] and involves a derivation of a differential equation (DE) for the envelope function of the cavity mode: 


\section{FThJ2.pdf}

$$
\left(-\frac{\omega_{L}}{C_{I}} \frac{d^{2}}{d x^{2}}+\omega_{L}^{2}-\omega^{2}\right) f(x)=\omega^{2} \int \delta \epsilon(x, y, z)\left\|\mathbf{E}_{k_{L}}(x, y, z)\right\|^{2} d y d z f(x)
$$

Here $\omega_{L}$ is the frequency of the band edge, $C_{L}$ is the band curvature and $\mathbf{E}_{k L}$ is the Bloch mode on the band edge. The coefficients of this DE then depend on the perturbation to the refractive index $\delta \varepsilon$ and on the properties of the band-edge modes, for which there exist a number of fast and efficient solvers. The splitting of the problem into the computation of the Bloch modes and the solution of a scalar DE results in an accurate method that is orders of magnitude faster than solving the complete problem: once the Bloch modes of the unperturbed structure are known a wide variety of perturbations may be explored very rapidly.

a)

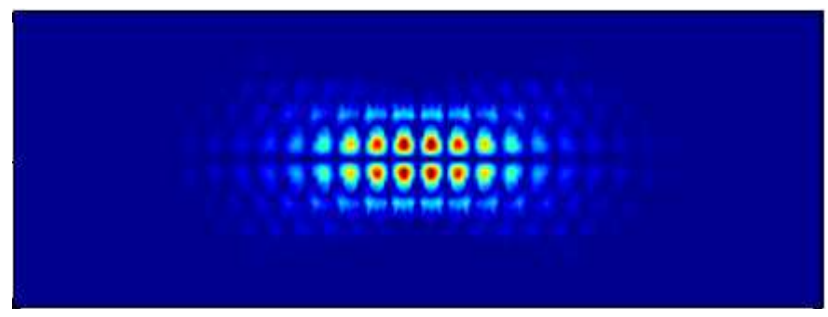

\author{
Figure 2: a) Comparison \\ between cavity modes computed \\ using direct numerical \\ computation with FDTD (top) \\ and our semi-analytical model \\ (bottom).The resonant \\ frequency is $d / \lambda=0.3160 \mathrm{~b}$ ) \\ Comparison showing the \\ computed envelope function for \\ the cavity mode. The resonant \\ frequency calculated from \\ perturbation theory is $d / \lambda=$ \\ 0.3158 . It should be noted that \\ there are no fitting parameters \\ in this computation.
}

b)

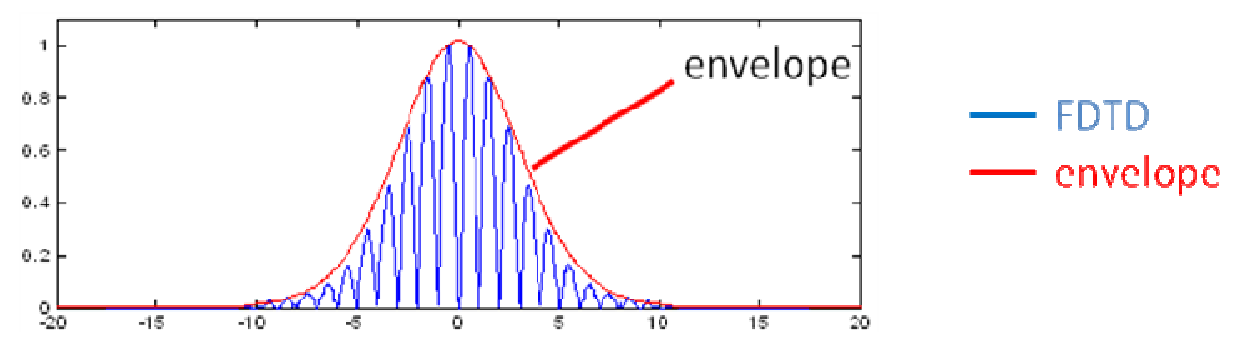

Using this method we are able to quickly and accurately characterize the envelope functions for 3D resonators (see Figure 2). We are also able to quantify both the resonant frequency and the degree of modal confinement as a function of perturbation strength, which will be very useful in evaluating candidates for optically-induced cavities. Because the method can be employed for an arbitrary perturbation, this allows us to optimize cavity designs using realistic computation times.

\section{References}

[1] B.S. Song, S. Noda and T. Asano, Science 300, Photonic Devices Based on In-Plane Hetero Photonic Crystals, 1537 (2003).

[2] B.S. Song, T.Asano, S. Noda, Y. Akahane, Ultra-high-Q photonic double-heterostructure nanocavity, Nature Materials 4, 207-210 (2005).

[3] S. Tomoljenovic-Hanic, M.J. Steel, C.M. de Sterke and D.J. Moss, High-Q cavities in photosensitive photonic crystals, Optics Letters 32, 542-544 (2007)

[4] J.M. Luttinger and W. Kohn, Motion of Electrons and Holes in Perturbed Periodic Fields, Phys. Rev. 97, 869-883 (1955). 